\title{
A NOTE ON RUSCHEWEYH TYPE OF INTEGRAL OPERATORS FOR UNIFORMLY $\alpha$-CONVEX FUNCTIONS
}

\author{
M. ANBU DURAI and R. PARVATHAM
}

Received 7 March 2001

We prove that the class of uniformly $\alpha$-convex functions introduced by Kanas is closed under the generalized Ruscheweyh integral operator for $0<\alpha \leq 1$.

2000 Mathematics Subject Classification: 30C45.

We denote by $\mathscr{A}$ the class of functions $f(z)=z+a_{2} z^{2}+\cdots$ which are analytic in $\Delta=\{z \in \mathbb{C}:|z|<1\}$. Let $S$ denote the class of functions in $\mathscr{A}$ that are univalent in $\Delta$. The subclasses of $S$ containing functions which are uniformly convex and uniformly starlike, introduced by Goodman [1, 2], are denoted by UCV and UST, respectively.

The class of uniformly $\alpha$-convex functions was introduced by Kanas [3] and she gave an analytic condition for such functions as follows: $f(z)$ is a uniformly $\alpha$-convex function if and only if

$$
\operatorname{Re}\left\{(1-\alpha) \frac{(z-\zeta) f^{\prime}(z)}{f(Z)-f(\zeta)}+\alpha\left(1+\frac{(z-\zeta) f^{\prime \prime}(z)}{f^{\prime}(z)}\right)\right\}>0
$$

for all $z, \zeta \in \Delta$ and $0 \leq \alpha \leq 1$. For $\zeta=0$, this class of functions reduces to Mocanu's class $M(\alpha)$ of $\alpha$-convex functions [4].

In this note, for $\alpha>0$, we consider the integral operator

$$
F(z)=\frac{F_{\alpha}(z, \zeta)-F_{\alpha}(0, \zeta)}{F_{\alpha}^{\prime}(0, \zeta)}
$$

where

$$
F_{\alpha}(z, \zeta)=\left\{\frac{c+1 / \alpha}{(z-\zeta)^{c}} \int_{\zeta}^{z}(t-\zeta)^{c-1}(f(t)-f(\zeta))^{1 / \alpha} d t\right\}^{\alpha}
$$

for all $z \in \Delta$ and for fixed $\zeta \in \Delta$ with $z \neq \zeta$. We prove that this normalized function $F(z)$ is a uniformly $\alpha$-convex function when $f(z)$ is a uniformly $\alpha$-convex function in the sense of Kanas [3].

For $\zeta=0$ the operator $F(z)$ reduces to Ruscheweyh's integral operator [5]. It is well known that Mocanu's class $M(\alpha)$ of $\alpha$-convex functions is closed under Ruscheweyh's integral operator for $\alpha>0$. 
THEOREM 1. Let $f(z)=z+a_{2} z^{2}+\cdots$ be a uniformly $\alpha$-convex function in $\Delta$ and let $c>0$. Then, for $0<\alpha \leq 1$, the function

$$
F(z)=\frac{F_{\alpha}(z, \zeta)-F_{\alpha}(0, \zeta)}{F_{\alpha}^{\prime}(0, \zeta)}, \quad z, \zeta \in \Delta,
$$

is uniformly $\alpha$-convex where $F_{\alpha}(z, \zeta)$ is defined as in (3).

Proof. We have from (3) that

$$
F_{\alpha}^{1 / \alpha}(z, \zeta)=\frac{c+1 / \alpha}{(z-\zeta)^{c}} \int_{\zeta}^{z}(t-\zeta)^{c-1}(f(t)-f(\zeta))^{1 / \alpha} d t
$$

Differentiating with respect to $z$, we have

$$
\begin{array}{r}
(z-\zeta)^{c} \frac{1}{\alpha} F_{\alpha}^{1 / \alpha-1}(z, \zeta) F_{\alpha}^{\prime}(z, \zeta)+c(z-\zeta)^{c-1} F_{\alpha}^{1 / \alpha}(z, \zeta) \\
=\left(c+\frac{1}{\alpha}\right)(z-\zeta)^{c-1}(f(z)-f(\zeta))^{1 / \alpha}
\end{array}
$$

and again differentiating with respect to $z$ we get

$$
\begin{aligned}
& \frac{1}{\alpha}\left\{(z-\zeta) F_{\alpha}^{1 / \alpha-1}(z, \zeta) F_{\alpha}^{\prime \prime}(z, \zeta)+(z-\zeta)\left(\frac{1}{\alpha}-1\right) F_{\alpha}^{1 / \alpha-2}(z, \zeta)\left(F_{\alpha}^{\prime}(z, \zeta)\right)^{2}\right. \\
& \left.\quad+F_{\alpha}^{1 / \alpha-1}(z, \zeta) F_{\alpha}^{\prime}(z, \zeta)\right\}+\frac{c}{\alpha} F_{\alpha}^{1 / \alpha-1}(z, \zeta) F_{\alpha}^{\prime}(z, \zeta) \\
& =\left(c+\frac{1}{\alpha}\right) \frac{1}{\alpha} f^{\prime}(z)(f(z)-f(\zeta))^{1 / \alpha-1} ; \\
& F_{\alpha}^{1 / \alpha-1}(z, \zeta) F_{\alpha}^{\prime}(z, \zeta)\left\{\alpha \frac{(z-\zeta) F_{\alpha}^{\prime \prime}(z, \zeta)}{F_{\alpha}^{\prime}(z, \zeta)}+(1-\alpha)(z-\zeta) \frac{F_{\alpha}^{\prime}(z, \zeta)}{F_{\alpha}(z, \zeta)}+\alpha(1+c)\right\} \\
& =(\alpha c+1) f^{\prime}(z)(f(z)-f(\zeta))^{1 / \alpha-1} .
\end{aligned}
$$

Thus we get

$$
\begin{aligned}
& F_{\alpha}^{1 / \alpha-1}(z, \zeta) F_{\alpha}^{\prime}(z, \zeta)\{\alpha {\left[1+\frac{(z-\zeta) F_{\alpha}^{\prime \prime}(z, \zeta)}{F_{\alpha}^{\prime}(z, \zeta)}-\frac{(z-\zeta) F_{\alpha}^{\prime}(z, \zeta)}{F_{\alpha}(z, \zeta)}\right] } \\
&\left.+\frac{(z-\zeta) F_{\alpha}^{\prime}(z, \zeta)}{F_{\alpha}(z, \zeta)}+c \alpha\right\} \\
&=(c \alpha+1) f^{\prime}(z)(f(z)-f(\zeta))^{1 / \alpha-1} .
\end{aligned}
$$

From (2) we have

$$
F^{\prime}(z)=\frac{F_{\alpha}^{\prime}(z, \zeta)}{F_{\alpha}^{\prime}(0, \zeta)}
$$

showing that $F(0)=0$ and $F^{\prime}(0)=1$.

Considering

$$
\frac{(z-\zeta) F^{\prime}(z)}{F(z)-F(\zeta)}=\frac{(z-\zeta) F_{\alpha}^{\prime}(z, \zeta)}{F_{\alpha}(z, \zeta)}
$$


and differentiating with respect to $z$, we have

$$
\begin{aligned}
\frac{F^{\prime \prime}(z)}{F^{\prime}(z)}+\frac{1}{z-\zeta}-\frac{F^{\prime}(z)}{F(z)-F(\zeta)} & =\frac{1}{z-\zeta}+\frac{F_{\alpha}^{\prime \prime}(z, \zeta)}{F_{\alpha}^{\prime}(z, \zeta)}-\frac{F_{\alpha}^{\prime}(z, \zeta)}{F_{\alpha}(z, \zeta)} \\
\frac{(z-\zeta) F^{\prime \prime}(z)}{F^{\prime}(z)}+1-\frac{(z-\zeta) F^{\prime}(Z)}{F(z)-F(\zeta)} & =1+\frac{(z-\zeta) F_{\alpha}^{\prime \prime}(z, \zeta)}{F_{\alpha}^{\prime}(z, \zeta)}-\frac{F_{\alpha}^{\prime}(z, \zeta)(z-\zeta)}{F_{\alpha}(z, \zeta)}
\end{aligned}
$$

Substituting (10) and (12) in (8), we obtain

$$
\begin{aligned}
& F_{\alpha}^{1 / \alpha-1}(z, \zeta) F_{\alpha}^{\prime}(z, \zeta)\left\{\alpha\left[\frac{(z-\zeta) F^{\prime \prime}(z)}{F^{\prime}(z)}+1-\frac{(z-\zeta) F^{\prime}(z)}{F(z)-F(\zeta)}\right]+\frac{(z-\zeta) F^{\prime}(z)}{F(z)-F(\zeta)}+c \alpha\right\} \\
& =(c \alpha+1) f^{\prime}(z)(f(z)-f(\zeta))^{1 / \alpha-1} ; \\
& F_{\alpha}^{1 / \alpha-1}(z, \zeta) F_{\alpha}^{\prime}(z, \zeta)\left\{(1-\alpha) \frac{(z-\zeta) F^{\prime}(z)}{F(z)-F(\zeta)}+\alpha\left(1+\frac{(z-\zeta) F^{\prime \prime}(z)}{F^{\prime}(z)}\right)+c \alpha\right\} \\
& =(c \alpha+1) f^{\prime}(z)(f(z)-f(\zeta))^{1 / \alpha-1} .
\end{aligned}
$$

Setting

$$
P(z, \zeta)=(1-\alpha) \frac{(z-\zeta) F^{\prime}(z)}{F(z)-F(\zeta)}+\alpha\left(\frac{(z-\zeta) F^{\prime \prime}(z)}{F^{\prime}(z)}+1\right)
$$

equation (13) becomes

$$
F_{\alpha}^{1 / \alpha-1}(z, \zeta) F_{\alpha}^{\prime}(z, \zeta)\{P(z, \zeta)+c \alpha\}=(c \alpha+1) f^{\prime}(z)(f(z)-f(\zeta))^{1 / \alpha-1}
$$

Taking logarithmic differentiation with respect to $z$, we get

$$
\begin{aligned}
& (1-\alpha)(z-\zeta) \frac{F_{\alpha}^{\prime}(z, \zeta)}{F_{\alpha}(z, \zeta)}+\alpha(z-\zeta) \frac{F_{\alpha}^{\prime \prime}(z, \zeta)}{F_{\alpha}^{\prime}(z, \zeta)}+\frac{\alpha(z-\zeta) P^{\prime}(z, \zeta)}{P(z, \zeta)+c \alpha}+\alpha \\
& =\alpha+\alpha \frac{(z-\zeta) f^{\prime \prime}(z)}{f^{\prime}(z)}+(1-\alpha) \frac{(z-\zeta) f^{\prime}(z)}{f(z)-f(\zeta)} \\
& \alpha\left[(z-\zeta) \frac{F_{\alpha}^{\prime \prime}(z, \zeta)}{F_{\alpha}^{\prime}(z, \zeta)}+1-\frac{(z-\zeta) F_{\alpha}^{\prime}(z, \zeta)}{F_{\alpha}(z, \zeta)}\right]+\frac{(z-\zeta) F_{\alpha}^{\prime}(z, \zeta)}{F_{\alpha}(z, \zeta)}+\frac{\alpha(z-\zeta) P^{\prime}(z, \zeta)}{P(z, \zeta)+c \alpha} \\
& =\alpha\left(1+\frac{(z-\zeta) f^{\prime \prime}(z)}{f^{\prime}(z)}\right)+(1-\alpha) \frac{(z-\zeta) f^{\prime}(z)}{f(z)-f(\zeta)} .
\end{aligned}
$$

Equations (10) and (12) give

$$
\begin{gathered}
\alpha\left[\frac{(z-\zeta) F^{\prime \prime}(z)}{F^{\prime}(z)}+1-\frac{(z-\zeta) F^{\prime}(z)}{F(z)-F(\zeta)}\right]+\frac{(z-\zeta) F^{\prime}(z)}{F(z)-F(\zeta)}+\frac{\alpha(z-\zeta) P^{\prime}(z, \zeta)}{P(z, \zeta)+c \alpha} \\
=\alpha\left(1+\frac{(z-\zeta) f^{\prime \prime}(z)}{f^{\prime}(z)}\right)+(1-\alpha) \frac{(z-\zeta) f^{\prime}(z)}{f(z)-f(\zeta)} .
\end{gathered}
$$


That is

$$
\begin{aligned}
& {\left[\alpha\left(1+\frac{(z-\zeta) F^{\prime \prime}(z)}{F^{\prime}(z)}\right)+(1-\alpha) \frac{(z-\zeta) F^{\prime}(z)}{F(z)-F(\zeta)}\right]+\frac{\alpha(z-\zeta) P^{\prime}(z, \zeta)}{P(z, \zeta)+c \alpha}} \\
& \quad=\alpha\left(1+\frac{(z-\zeta) f^{\prime \prime}(z)}{f^{\prime}(z)}\right)+(1-\alpha) \frac{(z-\zeta) f^{\prime}(z)}{f(z)-f(\zeta)} .
\end{aligned}
$$

Hence, we have

$$
P(z, \zeta)+\frac{\alpha(z-\zeta) P^{\prime}(z, \zeta)}{P(z, \zeta)+c \alpha}=\alpha\left(1+\frac{(z-\zeta) f^{\prime \prime}(z)}{f^{\prime}(z)}\right)+(1-\alpha) \frac{(z-\zeta) f^{\prime}(z)}{f(z)-f(\zeta)} .
$$

Since $f(z)$ is uniformly $\alpha$-convex, we have

$$
\operatorname{Re}\left\{P(z, \zeta)+\frac{\alpha(z-\zeta) P^{\prime}(z, \zeta)}{P(z, \zeta)+c \alpha}\right\}>0
$$

for all $z, \zeta \in \Delta, 0 \leq \alpha \leq 1$.

We show that $\operatorname{Re} P(z, \zeta)>0$. Suppose that there exists a point $\zeta_{0} \in \Delta$ such that the image of the arc $\Gamma: z(t)=\zeta_{0}+r e^{i t}$ is tangent to the imaginary axis. Let $w_{0}$ be the point of contact and let $z_{0} \in \Delta$ such that $w_{0}=P\left(z_{0}, \zeta_{0}\right)$. Then $\operatorname{Re} P\left(z_{0}, \zeta_{0}\right)=0$ and therefore $P\left(z_{0}, \zeta_{0}\right)=i x$, where $x \in R$. Hence the outer normal to $F(\Gamma)$ is

$$
\left(z_{0}-\zeta_{0}\right) P^{\prime}\left(z_{0}, \zeta_{0}\right)=y<0 .
$$

For such $\zeta_{0}$, we have

$$
\begin{aligned}
\operatorname{Re}\left\{P\left(z_{0}, \zeta_{0}\right)+\frac{\alpha\left(z_{0}-\zeta_{0}\right) P^{\prime}\left(z_{0}, \zeta_{0}\right)}{P\left(z_{0}, \zeta_{0}\right)+c \alpha}\right\} & =\operatorname{Re}\left\{i x+\frac{\alpha y}{c \alpha+i x}\right\} \\
& =\operatorname{Re}\left\{i x+\frac{\alpha y(c \alpha-i x)}{c^{2} \alpha^{2}+x^{2}}\right\} \\
& =\frac{c \alpha^{2} y}{(c \alpha)^{2}+x^{2}}<0 \text { for } c>0
\end{aligned}
$$

which contradicts (20) and hence $\operatorname{Re} P(z, \zeta)>0$ in $\Delta$ showing that $F(z)$ is a uniformly $\alpha$-convex function.

ACKNOWLEDGEMENT. This work was carried out when the first author was under the Faculty Improvement programme of University Grant and Commission of IX plan.

\section{REFERENCES}

[1] A. W. Goodman, On uniformly convex functions, Ann. Polon. Math. 56 (1991), no. 1, 87-92.

[2] _ On uniformly starlike functions, J. Math. Anal. Appl. 155 (1991), no. 2, 364-370.

[3] S. Kanas, Uniformly alpha convex functions, Int. J. Appl. Math. 1 (1999), no. 3, 305-310.

[4] P. T. Mocanu, Une propriété de convexité généralisée dans la théorie de la représentation conforme, Mathematica (Cluj) 11 (34) (1969), 127-133 (French).

[5] S. Ruscheweyh, Eine Invarianzeigenschaft der Basileviv Funktionen, Math. Z. 134 (1973), 215-219 (German).

M. Anbu Durai: The RAmanujan Institute for AdVANCED STUdy in Mathematics, UNIVERSITY OF MADRAS, CHENNAI-600 500, INDIA

E-mail address: anbuduraim@yahoo.co.in

R. Parvatham: The Ramanujan Institute for AdVAnced Study in Mathematics, UNIVERSITY OF MADRAS, CHENNAI-600 500, INDIA 


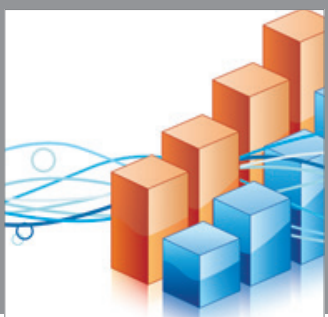

Advances in

Operations Research

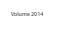

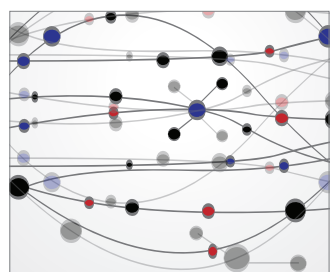

\section{The Scientific} World Journal
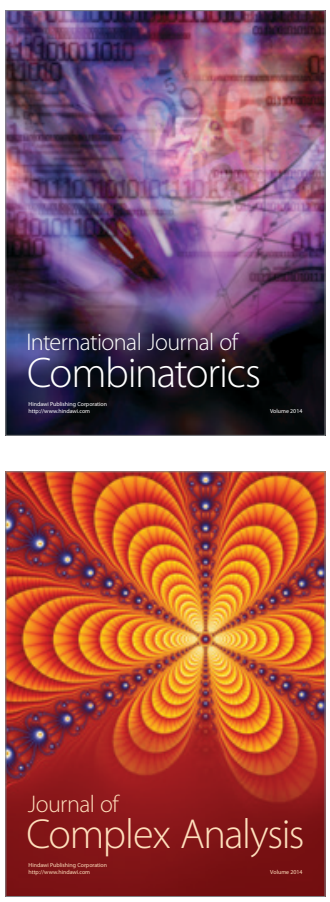

International Journal of

Mathematics and

Mathematical

Sciences
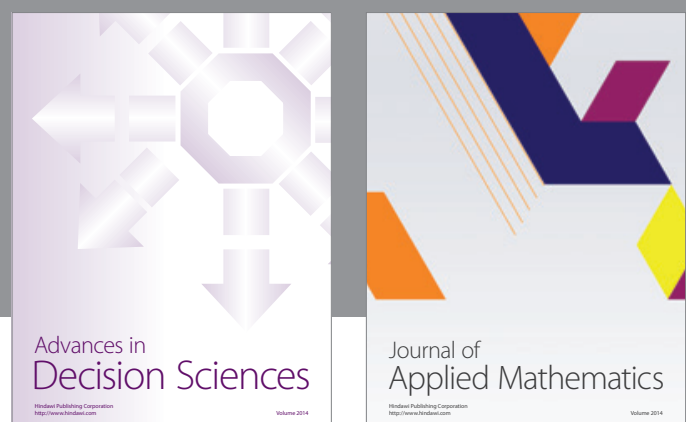

Journal of

Applied Mathematics
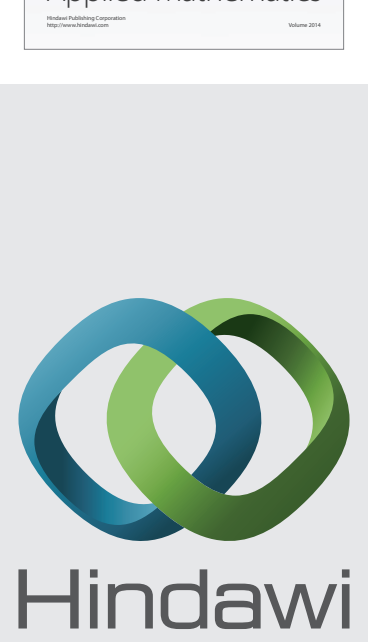

Submit your manuscripts at http://www.hindawi.com
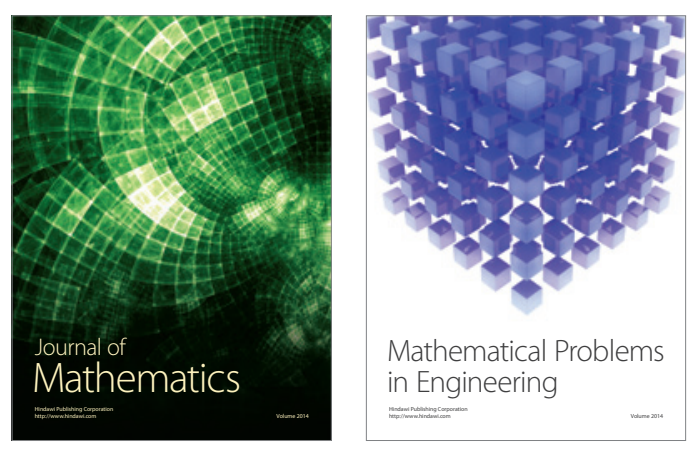

Mathematical Problems in Engineering
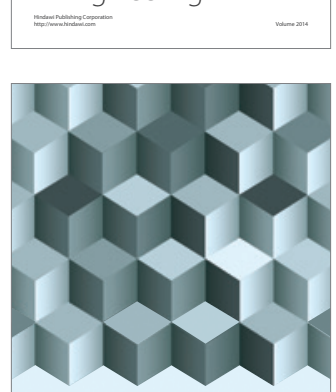

Journal of

Function Spaces
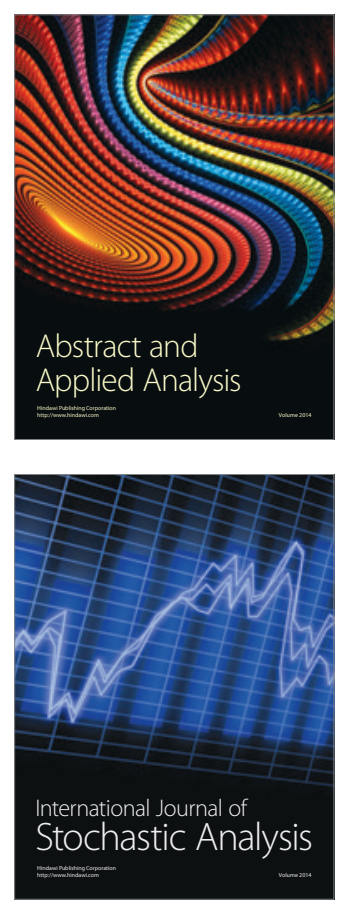

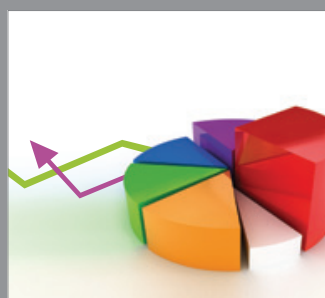

ournal of

Probability and Statistics

Promensencen
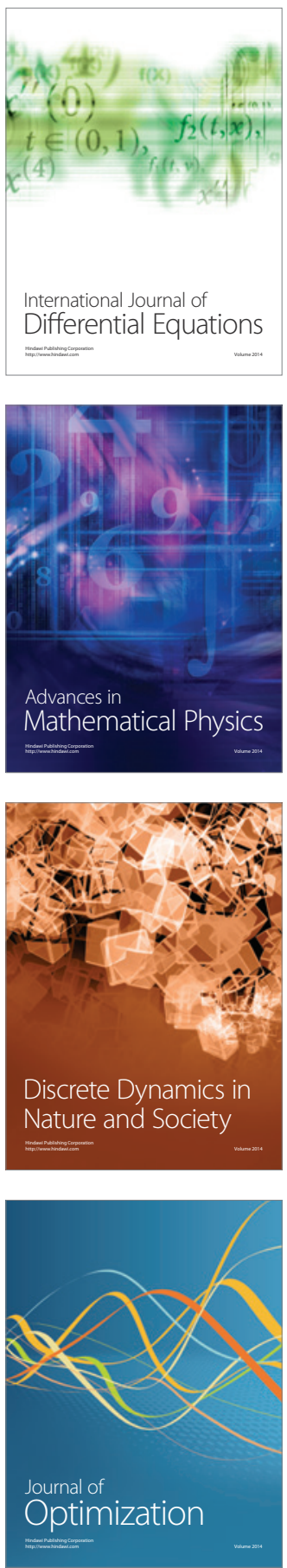